\title{
Emotional crisis in a naturalistic context: characterizing outpatient profiles and treatment effectiveness
}

\author{
Adriano Zanello ${ }^{1,3^{*}}$ (D) Laurent Berthoud ${ }^{2}$ and Jean-Pierre Bacchetta ${ }^{1}$
}

\begin{abstract}
Background: Crisis happens daily yet its understanding is often limited, even in the field of psychiatry. Indeed, a challenge is to assess the potential for change of patients so as to offer appropriate therapeutic interventions and enhance treatment program efficacy. This naturalistic study aimed to identify the socio-demographical characteristics and clinical profiles at admission of patients referred to a specialized Crisis Intervention Center (CIC) and to examine the effectiveness of the intervention.

Method: The sample was composed of 352 adult outpatients recruited among the referrals to the CIC. Assessment completed at admission and at discharge examined psychiatric symptoms, defense mechanisms, recovery styles and global functioning. The crisis intervention consisted in a psychodynamically oriented multimodal approach associated with medication.

Results: Regarding the clinical profiles at intake, patients were middle-aged $(M=38.56, S D=10.91)$, with a higher proportion of women (62.22\%). They were addressed to the CIC because they had attempted to commit suicide or had suicidal ideation or presented depressed mood related to interpersonal difficulties. No statistical differences were found between patients dropping out $(n=215)$ and those attending the crisis intervention $(n=137)$. Crisis intervention demonstrated a beneficial effect $(p<0.01)$ on almost all variables, with Effect Sizes (ES) ranging from small to large $(0.12<\mathrm{ES}<0.75$; median $=0.49)$. However, the Reliable Change Index indicated that most of the issues fall into the undetermined category (range 41.46 to $96.35 \%$; median $=66.20 \%$ ).

Conclusions: This study establishes the profile of patients referred to the $\mathrm{CIC}$ and shows that more than half of the patients dropped out from the crisis intervention before completion. Our findings suggest that people presenting an emotional crisis benefit from crisis intervention. However, given methodological constraints, these results need to be considered with caution. Moreover, the clinical significance of the improvements is not confirmed. Thus, the effectiveness of crisis intervention in naturalistic context is not fully determined and should be more rigorously studied in future research.
\end{abstract}

Keywords: Crisis intervention, Brief psychiatric rating scale, Defense mechanisms, Recovery style, Community mental health services, Treatment effectiveness

\footnotetext{
* Correspondence: adriano.zanello@hcuge.ch

${ }^{1}$ Department of Mental Health and Psychiatry, University Hospitals of Geneva,

Geneva, Switzerland

${ }^{3}$ HUG Département de Santé Mentale et de Psychiatrie, Site Belle-ldée, Ch.

du Petit-Bel-Air 2, CH-1225 Chêne-Bourg, Switzerland

Full list of author information is available at the end of the article
} 


\section{Background}

Crisis is a configuration involving a precipitating event, an emotional response and an intervention [1]. The nature of crisis events may be either public, as with natural disasters or terrorist attacks, or private, such as the notification of a chronic illness, domestic violence, loss of the sense of self, or sexual abuse [2]. Public crisis events are obvious, perceived and even shared by others, e.g. SARS crisis [3]. On the other hand, private events are often hidden to others who may be unaware of what is going on [4]. The way people perceive and experience the event may lead to a crisis response. The latter is an individual reaction to stress with the potential to produce psychopathological symptoms [5]. It is also considered as a transition phase that may change behaviors and interpersonal functioning [6]. Crisis interventions are thought to provide acute psychological care enabling people in crisis to recover their adaptive level of functioning and to circumvent potential deleterious effects of the psychological trauma [7].

Among crisis interventions one can find psychodynamic, behavioral, cognitive-behavioral, developmental, systems, radical and social construction approaches as well as eye movement desensitization and reprocessing (EMDR) and strategic solution-focused therapy, crisis incident and stress management for specific crises $[4,7,8]$. These treatments are delivered generally during 2 months by well-trained staff members (e.g. psychiatrists, nurses, psychologists, social workers...) in specific structures such as Crisis Resolution Teams [9, 10], Women's Crisis Houses [11], Crisis Intervention Units [12] or Crisis Intervention Centers [13-17]. Even though there are important differences among services and clinical practices, crisis interventions share some characteristics - such as administering rapid and timelimited short-term interventions ( $<3$ months), being a $24 / 7$ service, offering a helpline - that provide an alternative to psychiatric hospitalization, facilitate discharge, play a gatekeeping function regarding inpatient units, involve multidisciplinary teams, and aim to stabilize individuals facing a crisis. Crisis interventions also share common ingredients in treatment success, namely "therapist warmth, and empathy, the therapeutic alliance, insight and feedback and action factors such as cognitive mastery" (p. 18) [2]. One of the challenges in crisis intervention is to seize the potential for change while giving patients enough support and security as well as maintaining social integration [15]. Therefore, it is important to carefully identify the patient's characteristics at admission in specific crisis structures, in order to offer appropriate therapeutic interventions and improve the efficacy of the treatment programs [18]. On the other hand, it is also crucial, for patients, relatives, caregivers, hospital policy, and social insurances to document crisis interventions efficiency. Several studies have examined the efficiency of the specific treatments administered in various crisis center contexts. Most of the research examined whether Crisis Intervention contributed to reducing the global rate of psychiatric hospitalization and readmission in emergency units to cost-effectiveness, and enhanced client satisfaction $[9,10,15,16,19-22]$. A meta-analysis comprised of 36 crisis intervention studies yielded large effect sizes [2]. Unfortunately, the authors did not specify the measures included. Only few studies have reported Crisis Intervention effects on symptoms, social functioning and quality of life, showing a positive short term effect on these variables [23-25], while in the long term findings remain controversial [14].

Several authors have claimed that the central characteristic of crisis is its potential for change in the psychological functioning of patients $[6,13,26-28]$. Moreover, within the framework of psychodynamic theory, it is hypothesized that patients preferentially use maladaptive defense mechanisms during emotional crisis [27]. To the best of our knowledge, no empirical study has examined these aspects of crisis. Thus, it seems that "... research on crisis intervention is in its early stage of development" (p.18) [2].

This led us to design a clinical prospective naturalistic study, which is more suitable to complex interventions, such as those taking place in the CICs, rather than randomized research $[29,30]$. The aims of this study were a) to present the functioning of a $\mathrm{CIC}, \mathrm{b})$ to characterize the crisis context, socio-demographic, psychiatric and clinical profile of the patients referred to the $\mathrm{CIC}$ and c) to assess the effectiveness of a psychodynamically oriented [27] crisis intervention.

\section{Methods}

In Geneva, the CIC was introduced as an alternative to hospitalization [13] as part of psychiatry sectorization. Referrals to the $\mathrm{CIC}$ are made by the patients themselves or by concerned others such as private practitioners (e.g. psychiatrists, psychotherapists, physicians...), the emergency unit of the General Hospital, specialized mental units (e.g. affective disorders, eating disorders, personality disorders, substances abuse units...), and outpatient and inpatient units of the Psychiatric Hospital.

The CIC team is multidisciplinary. It offers three main services: clinical assessment, time-limited support therapy, and crisis intervention either in individual or group format. If necessary, patients can be offered one of the eight crisis beds in the center for up to seven nights.

Admission to the $\mathrm{CIC}$ is done in the $24 \mathrm{~h}$ after the initial call. Patients participating in the crisis program are assigned to a psychiatrist and a nurse; this duo remains stable throughout the intervention. Patients participating in the crisis intervention are proposed individual and group therapy as well as support by social workers, and 
family or couple interventions [15]. Crisis intervention generally lasts $6-8$ weeks [27], but its length can be tailored to the patient's specific needs. The assigned duo decides with the patient when to end the program.

\section{Patients}

Outpatients were recruited among the referrals to one of the four CICs of the Department of Psychiatry, which is part of the Geneva University Hospitals in Switzerland, from 2005 to 2010 . Inclusion criteria were: being 18 to 65 years old, fulfilling an ICD-10 diagnosis for mental disorders, presenting an emotional crisis, and participating in the intensive crisis intervention program [15]. Exclusion criteria were: difficulties in spoken or written French, behaviors that could not allow assessment (e.g. hostility, disorganization, under the influence of substance abuse), suspicion of or documented neurocognitive alterations, or mental retardation.

\section{Measures}

Measures at admission included socio-demographic information, crisis details and clinical variables. Patients participated in a semi-structured interview assessing psychiatric symptom severity and completed a set of self-administered questionnaires measuring psychiatric symptoms, defense mechanisms, recovery styles and global functioning.

\section{Symptoms}

Psychiatric symptoms were assessed with two tools, the Brief Psychiatric Rating Scale expanded version 4.0 (BPRS 4.0) [31] (Zanello et al., Adaptation française de la Brief Psychiatric Rating Scale version 4.0, unpublished) and the Symptom Checklist 90 - Revised (SCL-90-R) [32, 33]. The BPRS 4.0 is a semi-directive interview composed of 24 items rated from 1 (absent) to 7 (severe). In order to have a general representation of symptom severity, and because no calibration of the BPRS 4.0 to equivalent clinical judgments exists, we borrowed and modified the categories defined by Brenner's group [34]. We considered at intake five categories of general distress defined as follows: no illness (all items scores $<3$ ), mild severity (at least one item $=3$, but no item $\geq 4$ ), moderate (at least one item $=4$, but no item $\geq 5$ ), severe (at least one item $=5$, but no item $\geq 6$ ), and extremely severe (at least one item $\geq 6$ ). This procedure was used because low BPRS 4.0 scores can be obtained even if only one item is rated extremely severe (score $=7$ ) while the others are absent (score $=1$ ). This variable was labeled BPRS-4.0 Categories (BPRS 4.0-C). Changes occurring after $\mathrm{CIC}$ intervention were examined comparing the BPRS 4.0 mean score of reality distortion, apathy, activity, mood disturbance, disorganization and somatization dimensions [35], obtained at intake and at discharge.
The SCL-90-R is a self-rating scale composed of 90 items rated from 0 (not at all) to 4 (extremely). At intake, we considered the Global Severity Index (GSI) and transformed it into T-scores (mean $=50, \mathrm{SD}=10$ ) found in published norms [36]. Borrowing the guidelines for interpreting T scores [37], we considered five categories defined as follows: no distress $(T \leq 55)$, mild $(56 \leq T<60)$, moderate $(61 \leq T<65)$, severe $(65 \leq T<70)$, and extremely severe $(T \geq 70)$. This variable was labelled SCL-90-R Categories (SCL-90-R-C). Possible changes due to crisis intervention were examined comparing the scores at intake and at discharge on the nine symptoms dimensions of SCL-90-R: Somatisation, Obsessive-compulsivity, Interpersonal sensitivity, Depression, Anxiety, Hostility, Phobic anxiety, Paranoid ideation and Psychoticism.

\section{Defense mechanisms and recovery styles}

As emotional crisis leads to a breakdown in defense mechanisms [27], the latter were examined with the Defense Style Questionnaire (DSQ 40) [38, 39]. The DSQ 40 is a self-reported questionnaire composed of 40 items assessed on a 9-point Likert scale. It measures 20 defense mechanisms, each being represented by 2 items. These mechanisms are grouped as being adaptive (mature defense: e.g. humor, suppression, sublimation), intermediate (neurotic defense: e.g. undoing, idealization, reaction formation), or maladaptive (immature defense: e.g. splitting, projection, acting out). A mean score was calculated for each of these 3 dimensions.

The Recovery Style Questionnaire (RSQ) [40] (Zanello and Koellner, Adaptation française du Recovery Style Questionnaire, unpublished) assesses the patients' attitude about their mental illness. This attitude is underpinned by the mechanisms of defense. The RSQ is a self-reported questionnaire composed of 39 yes-no items. It assesses on a continuum two opposite main recovery styles identified as integration and sealing-over [41]. The integration style characterized patients curious about their crisis, recognizing continuity in their mental life before, during and after the crisis, which is considered as a positive experience for the future. The integration style is associated to more mature defense mechanisms. On the other hand, the sealingover style characterized patients viewing their crisis as an interruption in their mental life, not related to their personal difficulties and as an unworthy experience to examine. The sealing-over style is related to maladaptive mechanisms of defense. A dimensional score ranging from 0 to 100 was calculated [42]. Scores higher than 49 reflect "integration"; while scores equal to or lower than 49 indicate "sealing-over" of the emotional crisis.

\section{General functioning}

The Global Assessment Functioning (GAF) scale describes the current psychological, social and occupational 
functional status of a patient in a single measure ranging from 0 to 100 [43] with higher scores reflecting better functioning and low symptomatology.

\section{Procedure}

The overseeing institutional Ethics Committee approved the study. The study was completely described to patients who gave their informed written consent before assessment. Clinical psychologists performed the assessment. All of them received formal training in using the BPRS 4.0 [35]. Inter-rater reliability was considered excellent (intraclass coefficient correlation $>0.80$ ). Assessments were administered as soon as possible at admission within the first 2 weeks of CIC treatment. To address the effectiveness of crisis intervention, all the patients who gave formal consent were invited to fulfill the assessment once again at discharge. It is also to be mentioned that the DSQ-40 was not included in the initial study protocol and was the object of an addendum that took time to be examined before being accepted.

\section{Statistical analysis}

The normality of the distribution of the variables was verified with the Kolmogorov-Smirnov test. The results suggested violation of the normality for all variables; we thus analyzed the data using non-parametric statistics. Group differences (e.g. gender, diagnosis) were examined with Friedman analyses of variance, the Wilcoxon test for related samples, and the Mann-Whitney U-test for independent samples. Association between variables was computed with Spearman's rho correlation. Categorical variables were analyzed with the Pearson Chi-Square. Statistical analyses were performed with SPSS version 22.0. The significance level was set at $p \leq 0.05$ after Bonferroni correction for multiple comparisons in order to minimize the risk of false results. The effect size (ES) was estimated using $r\left(r=z / n^{1 / 2}\right)$ approximate value [44] and interpreted according to usual guidelines [45], where ES is small when $\leq 0.20$, medium when $\leq 0.50$ and large when $\leq 0.80$. As inferential statistical analyses and ES only give an incomplete picture of the effect of crisis intervention, the Reliable Change Index (RCI) [46] was also calculated. This enabled to verify whether changes were due to crisis intervention instead of measure errors. The RCI for each patient and each single variable was calculated correcting for measure error and practice effect. The RCI was calculated with the following formula $\mathrm{RCI}=[(\mathrm{X} 1-\mathrm{X} 2) / \mathrm{SE}]$, where $\mathrm{X} 1$ and $\mathrm{X} 2$ represent patient scores at intake and discharge respectively, and SE $($ standard error $)=\mathrm{SD} 1 *(1-\mathrm{r})^{1 / 2}$, where SD1 $=$ standard deviation of the variable at intake and $r=$ test - retest reliability computed with non-parametric correlation coefficients (Spearman's rho or Cronbach's alpha). To classify outcome as improved or deteriorated we chose a criterion of RCI $\pm|1.96|$ with at least a $95 \%$ confidence interval; when this criterion was not met outcome was considered as undetermined. The outcome of each variable was classified according to these three categories. As recommended in the literature [47, 48], we also reported the proportion of missing data and the percentage of dropouts. In order to examine crisis intervention effectiveness with the maximum of data, we handled the problem of missing data using all the observations per variable only if the patient was administered the BPRS 4.0 at intake and at discharge. The main reason legitimating this choice was the fact that we were studying the psychometric characteristics of the BPRS 4.0 in a sample of patients presenting an emotional crisis [35]. We were also interested in testing whether "Non Attenders" (patients participating only to initial assessment and crisis intervention) differed from "Attenders" (patients participating to both assessments and crisis intervention) because significant differences between these two groups may underpower statistical analyses, constitute a bias in sample selection, and prevent generalization of crisis intervention effectiveness [49].

\section{Results}

\section{Characteristics of the sample at intake}

Baseline assessments (T0) were computed within 12.12 days on average $(\mathrm{SD}=11.22)$ after intake the initial appointment with the psychiatrist and the nurse.

\section{Socio-demographic variables}

As presented in Table 1, this study included 352 middle-aged patients $($ mean $=38.56, \mathrm{SD}=10.91)$, two thirds being women (62.22\%), the latter being younger than the men. No other gender differences were found. Only few patients $(n=7,1.99 \%)$ referred to the CIC lived in sheltered housings or benefited from accommodations in hotels. Almost two thirds were employed or students while the unemployed benefited from the financial support of social insurances (e.g. social security, unemployment pension or disability pension).

\section{Crisis context}

As shown in Table 2, patients were mainly referred by the emergency unit. The most common symptoms motivating admission were suicidal ideation or suicide attempt, depressed mood, and anxiety. The predominant trigger events of crisis were couple difficulties, work and family relations. It is to be noted that the trigger event was difficult to categorize or unclear for $20 \%$ of patients. Women presented more family problems, couple difficulties, and unclear triggers, while men showed more triggers falling under the category labeled "others" (e.g. 
Table 1 Socio-demographic characteristics of the sample $(N=352)$

\begin{tabular}{|c|c|c|c|c|c|c|c|c|}
\hline & \multicolumn{2}{|c|}{ Sample $(N=352)$} & \multicolumn{2}{|c|}{ Women $(N=219)$} & \multicolumn{2}{|c|}{ Men $(N=133)$} & \multirow[t]{2}{*}{ Statistics } & \multirow[t]{2}{*}{$p$} \\
\hline & Mean & SD & Mean & SD & Mean & SD & & \\
\hline \multirow[t]{2}{*}{ Age } & 38.56 & 10.91 & 37.41 & 11.29 & 40.45 & 10.00 & $Z=-2.69$ & 0.007 \\
\hline & N & (\%) & $\mathrm{N}$ & (\%) & $\mathrm{N}$ & (\%) & & \\
\hline \multicolumn{9}{|l|}{ Living conditions $^{\mathrm{a}}$} \\
\hline Own flat, family, and shared accommodation & 341 & $(98.00)$ & 214 & $(61.50)$ & 127 & $(36.50)$ & $x^{2}=1.16(d f=1)$ & 0.28 \\
\hline Sheltered housing & 7 & $(2.01)$ & 3 & $(0.86)$ & 4 & $(1.14)$ & & \\
\hline \multicolumn{9}{|l|}{ Occupational status ${ }^{\mathrm{b}}$} \\
\hline Employed/student & 223 & $(66.35)$ & 139 & (41.49) & 84 & $(25.07)$ & $X^{2}=0.08(d f=1)$ & 0.77 \\
\hline Unemployed & 112 & (36.65) & 68 & (20.29) & 44 & (13.13) & & \\
\hline
\end{tabular}

Missing data: ${ }^{\mathrm{a}} \mathrm{N}=4$ and ${ }^{\mathrm{b}} \mathrm{N}=17$

domestic violence, alcohol abuse...). No other gender difference was found.

\section{Main diagnosis and medication}

Table 3 summarizes patients' diagnosis and medication status. The most common main diagnosis was mood disorders $(n=253,72.16 \%)$. The next important category of diagnoses was neurotic, stress-related and somatoform disorders ( $n=76,21.59 \%)$. Other diagnoses represented altogether $6.53 \%(n=23)$. No gender differences were found.

Most of the patients $(n=320,90.90 \%)$ received psychotropic medication with the majority taking more than one drug. As principal medication, patients were administered antidepressants $(n=213,60.51 \%)$, antipsychotics ( $n=44,12.50 \%)$, hypnotics ( $n=15,4.26 \%)$, mood stabilizers

Table 2 Crisis context at admission for the sample, by gender, and results of gender comparison

\begin{tabular}{|c|c|c|c|c|c|c|c|c|}
\hline & Samp & & Wom & & Mer & & Statistics & $p$ \\
\hline & $\mathrm{N}$ & (\%) & $\mathrm{N}$ & (\%) & $\bar{N}$ & (\%) & & \\
\hline Referred by & & & & & & & $x^{2}=7.72(d f=5)$ & 0.10 \\
\hline Emergency unit & 164 & $(46.59)$ & 109 & $(30.97)$ & 55 & $(15.63)$ & & \\
\hline Outpatient unit & 33 & $(9.38)$ & 21 & $(5.97)$ & 12 & $(3.41)$ & & \\
\hline Private practitioner & 106 & $(30.11)$ & 67 & $(19.03)$ & 39 & $(11.08)$ & & \\
\hline Psychiatric hospital & 39 & (11.08) & 17 & $(4.83)$ & 22 & $(6.25)$ & & \\
\hline Self-referred & 10 & $(2.84)$ & 5 & $(1.42)$ & 5 & $(1.42)$ & & \\
\hline Specialized psychiatric units ${ }^{a}$ & 0 & $(0.00)$ & 0 & $(0.00)$ & 0 & $(0.00)$ & & \\
\hline Primary problem presented & & & & & & & $x^{2}=8.11(d f=7)$ & 0.32 \\
\hline Anxiety & 50 & $(14.20)$ & 28 & (7.95) & 22 & $(6.25)$ & & \\
\hline Delusions/hallucinations & 13 & (3.93) & 6 & $(17.04)$ & 7 & $(1.99)$ & & \\
\hline Depressed mood & 112 & $(31.82)$ & 71 & $(20.17)$ & 41 & $(11.64)$ & & \\
\hline Substance abuse & 7 & $(1.99)$ & 5 & $(1.42)$ & 2 & $(0.57)$ & & \\
\hline Suicidal ideation or attempt & 143 & $(40.63)$ & 92 & $(26.13)$ & 51 & $(14.49)$ & & \\
\hline Violence & 5 & $(1.42)$ & 1 & $(0.28)$ & 4 & $(1.14)$ & & \\
\hline Not reported & 17 & $(4.83)$ & 13 & (3.69) & 4 & $(1.14)$ & & \\
\hline Other & 5 & $(1.42)$ & 2 & $(0.57)$ & 3 & $(0.85)$ & & \\
\hline Trigger event & & & & & & & $x^{2}=25.75(d f=5)$ & $<0.001$ \\
\hline Couple & 148 & $(42.05)$ & 101 & $(28.69)$ & 47 & $(13.35)$ & & \\
\hline Family & 59 & $(16.76)$ & 44 & $(12.50)$ & 15 & $(4.26)$ & & \\
\hline Work & 69 & $(19.60)$ & 38 & $(10.79)$ & 31 & $(8.81)$ & & \\
\hline Other & 53 & $(15.06)$ & 20 & (5.68) & 33 & (9.38) & & \\
\hline Unclear & 23 & (6.53) & 16 & $(4.54)$ & 7 & (1.99) & & \\
\hline
\end{tabular}

${ }^{a}$ Given $\mathrm{N}$ values this Group was excluded of $\mathrm{X}^{2}$ analyses 
Table 3 Main diagnosis and medication of the sample $(N=352)$

\begin{tabular}{|c|c|c|c|c|c|c|c|c|}
\hline & \multicolumn{2}{|c|}{ Sample } & \multicolumn{2}{|c|}{ Women } & \multicolumn{2}{|c|}{ Men } & \multirow[t]{2}{*}{ Statistics } & \multirow[t]{2}{*}{$p$} \\
\hline & $\bar{N}$ & (\%) & $\bar{N}$ & (\%) & $\bar{N}$ & (\%) & & \\
\hline \multicolumn{9}{|l|}{ ICD-10 Diagnosis ${ }^{\mathrm{a}}$} \\
\hline F30-39: Mood (affective) disorders & 253 & $(72.16)$ & 155 & $(44.03)$ & 98 & $(27.84)$ & $x^{2}=5.12(d f=4)$ & 0.28 \\
\hline F31: Bipolar affective disorder & 18 & $(5.11)$ & & & & & & \\
\hline F32: Depressive episode & 131 & $(37.22)$ & & & & & & \\
\hline F33: Recurrent depressive disorder & 104 & $(29.54)$ & & & & & & \\
\hline F40-F48 Neurotic, stress-related and somatoform disorders & 76 & $(21.59)$ & 50 & $(14.20)$ & 26 & (7.38) & & \\
\hline F40: Phobic anxiety disorders & 4 & $(1.13)$ & & & & & & \\
\hline F41: Other anxiety disorders & 9 & $(2.56)$ & & & & & & \\
\hline F42: Obsessive compulsive disorder & 3 & $(8.52)$ & & & & & & \\
\hline F43: Reaction to severe stress & 60 & $(17.05)$ & & & & & & \\
\hline Others & 23 & $(6.53)$ & 14 & (3.98) & 9 & $(2.56)$ & & \\
\hline $\begin{array}{l}\text { F10 Mental and behavioral disorders due to psychoactive } \\
\text { substance use }\end{array}$ & 3 & $(0.85)$ & & & & & & \\
\hline F20 Schizophrenia, schizotypal and delusional disorders; & 5 & $(1.42)$ & & & & & & \\
\hline $\begin{array}{l}\text { F50 Behavioral syndromes associated with physiological } \\
\text { disturbances and physical factors }\end{array}$ & 3 & $(0.56)$ & & & & & & \\
\hline F60 Specific personality disorders & 11 & $(3.13)$ & & & & & & \\
\hline Z73 Problems related to life - management difficulties & 1 & $(0.03)$ & & & & & & \\
\hline \multicolumn{9}{|l|}{ Medication } \\
\hline None & 32 & $(9.10)$ & 20 & $(5.68)$ & 12 & $(3.40)$ & $x^{2}=1.35(d f=2)$ & 0.51 \\
\hline Monotherapy & 61 & (17.33) & 34 & $(9.65)$ & 27 & $(7.67)$ & & \\
\hline Polytherapy & 259 & (73.58) & 165 & $(46.87)$ & 94 & (26.70) & & \\
\hline
\end{tabular}

${ }^{\mathrm{a} A s}$ the frequency for number of cells was very low for women and men (not reported), statistics were compute considering the following three categories: F30-39: Mood (Affective) Disorders, F40-F48 Neurotic, stress-related somatoform disorders and Others

( $n=14,3.97 \%)$, and benzodiazepines ( $n=11,3.13 \%)$. The other psychotropic medications were administered for less than $1 \%$ of the sample. No gender differences were found.

\section{Clinical characteristics}

Table 4 contains details about the clinical psychiatric features of the sample. Most patients presented severe to extremely severe psychiatric symptoms on both the BPRS 4.0-C and the SCL-90-R-C. These two measures were positively and significantly correlated $(r=.39$, $p<0.001)$. The patients used their defense mechanisms differentially (Friedman $X^{2}=37.45$, df $=2, p<0.001$ ). Post-hoc analyses show that mature defenses were more present than neurotic (Wilcoxon $\mathrm{Z}=-3.68, p<0.001$ ) and immature defenses (Wilcoxon $\mathrm{Z}=-6.68, p<0.001$ ), and neurotic defenses were more present than immature defenses (Wilcoxon $\mathrm{Z}=-4.05, p<0.001$ ). One fifth of the patients showed a sealing-over recovery style to overcome their emotional crisis. The GAF score indicates that patients presented, on average, moderate symptoms of mental illness or moderate functional impairments. None of the comparisons between genders reached statistical significance.

\section{Crisis intervention effectiveness Dropouts}

Among the 352 patients, 58.5\% skipped the assessment at discharge. Attenders were older (mean $=40.65$ years, $\mathrm{SD}=11.47$ ) than Non Attenders (mean $=37.08$ years; $\mathrm{SD}=10.26)$ (Mann-Whitney $\mathrm{Z}=-2.81, p=0.005)$. The proportion of men and women was equivalent in both groups ( $\left.\chi^{2}=0.73, \mathrm{df}=1, p=0.39\right)$. Regarding "activity and incomes", Attenders depended less on the social support system $\left(\chi^{2}=14.7, \mathrm{df}=5, p=0.01\right)$. They also presented more obsessive features in SCL-90-R dimensions (Attenders: mean $=1.82, \mathrm{SD}=0.83$; Non Attenders: mean $=1.61, \mathrm{SD}=0.82$, Mann-Whitney $\mathrm{Z}=-2.28$, $p=0.02)$. No other difference reached statistical significance. Also there was no difference in medication use $\left(\chi^{2}=1.35, \mathrm{df}=2, p=0.58\right)$. Psychopathology and social functioning were similar in both groups (all $p>0.10$; for details see Additional file 1). 
Table 4 Psychiatric symptoms, defense mechanisms, recovery styles and social functioning

\begin{tabular}{|c|c|c|c|c|c|c|c|c|c|}
\hline \multirow[t]{2}{*}{ Variables } & & \multicolumn{2}{|c|}{ Sample } & \multicolumn{2}{|c|}{ Women } & \multicolumn{2}{|l|}{ Men } & \multirow[t]{2}{*}{ Statistics } & \multirow[t]{2}{*}{$p$} \\
\hline & & $\mathrm{N}$ & $(\%)$ & $\mathrm{N}$ & $(\%)$ & $\mathrm{N}$ & (\%) & & \\
\hline \multicolumn{10}{|c|}{ Psychiatric symptoms } \\
\hline \multirow[t]{5}{*}{ BPRS-4.0 } & No illness ${ }^{a}$ & 0 & $(0.00)$ & 0 & $(0.00)$ & 0 & $(0.00)$ & $x^{2}=0.65(d f=3)$ & 0.89 \\
\hline & Mild & 6 & $(1.77)$ & 4 & $(1.17)$ & 2 & $(0.58)$ & & \\
\hline & Moderate & 27 & (7.95) & 15 & $(4.41)$ & 12 & $(3.52)$ & & \\
\hline & Severe & 71 & (20.88) & 44 & $(12.94)$ & 27 & $(7.94)$ & & \\
\hline & Extremely severe & 236 & $(69.47)$ & 149 & $(43.82)$ & 87 & $(25.58)$ & & \\
\hline \multirow[t]{5}{*}{ SCL-90-R } & No distress & 10 & $(3.65)$ & 4 & $(1.45)$ & 6 & $(2.18)$ & $x^{2}=5.92(d f=4)$ & 0.21 \\
\hline & Mild & 8 & $(2.92)$ & 6 & $(2.18)$ & 2 & $(0.73)$ & & \\
\hline & Moderate & 16 & $(5.84)$ & 8 & $(2.92)$ & 8 & $(2.92)$ & & \\
\hline & Severe & 32 & $(11.67)$ & 24 & $(8.76)$ & 8 & $(2.92)$ & & \\
\hline & Extremely severe & 208 & (75.91) & 132 & $(48.17)$ & 76 & $(27.73)$ & & \\
\hline \multirow[t]{3}{*}{ Recovery (RSQ) } & Sealing over & 54 & (19.01) & 39 & (13.73) & 15 & $(5.28)$ & $x^{2}=2.78(d f=1)$ & 0.10 \\
\hline & Integration & 230 & (80.98) & 138 & $(48.59)$ & 92 & (32.39) & & \\
\hline & & Mean & SD & Mean & SD & Mean & SD & & \\
\hline \multirow[t]{3}{*}{ Defenses (DSQ-40) } & Mature & 5.24 & 1.31 & 5.21 & 1.32 & 5.28 & 1.32 & $Z=-0.25$ & 0.80 \\
\hline & Neurotic & 4.66 & 1.41 & 4.67 & 1.45 & 4.65 & 1.32 & $Z=-0.11$ & 0.92 \\
\hline & Immature & 4.24 & 1.07 & 4.18 & 1.04 & 4.32 & 1.11 & $Z=-0.54$ & 0.59 \\
\hline \multicolumn{2}{|c|}{ Global functioning (GAF) } & 52.51 & 10.99 & 52.56 & 11.21 & 52.42 & 10.64 & $Z=-0.58$ & 0.57 \\
\hline
\end{tabular}

BPRS-4.0 Brief Psychiatric Rating Scale version 4.0, SCL-90-R Symptom Checklist - Revised, DSQ-40 Defense Style Questionnaire 40 items, RSQ Recovery Style Questionnaire, GAF Global Assessment Functioning

${ }^{a}$ Given $N$ values this Group was excluded of $X^{2}$ analyses

\section{Missing data at discharge}

Table 5 also provides the number of observations per variable. The highest proportion of missing data at discharge was observed for the DSQ-40 (48.18\%), followed by the GAF (22.62\%), the RSQ (21.16\%) and the SCL-90-R (10.22\%).

\section{Length of intervention}

The crisis intervention lasted on average 57 days (median) [ minimum $=11$; maximum $=409$ days $]$.

\section{Intake vs discharge results comparisons}

Table 5 summarizes the statistical results of the comparisons. After crisis intervention there was a significant reduction of symptom severity on the BPRS 4.0 and SCL-90-R dimensions, except for the Activation dimension of the BPRS 4.0. There was also a significant decrease of immature defense mechanisms and a greater use of mature mechanisms, whereas neurotic defenses remained stable (DSQ-40). Patients adopted a more integrative recovery style (RSQ) and had a better global functioning (GAF). All the comparisons survived the conservative Bonferroni correction for multiple comparisons, except the apathy and disorganization BPRS 4.0 factors and the mature defense mechanisms (DSQ-40). None of the improvements were related to gender (all $p>0.10$ ).

\section{Effect sizes}

As reported in Table 5, the crisis intervention had medium effect sizes for all variables except for the Activation and Disorganization dimensions of the BPRS 4.0 where the magnitude of the effect size was small. Also to be mentioned, the highest magnitude was found in observer mood disturbance and depression variables.

\section{Reliability Change Index ( $R C I)$}

Table 6 summarizes the RCI for each variable. After crisis intervention, the clinical evolution fell into the undetermined category for most patients, with proportions ranging from $41.46 \%$ to $96.35 \%$; some patients clinically deteriorated, with proportions ranging from $0.81 \%$ to $18.31 \%$ and some improved with proportions ranging from $2.19 \%$ to $52.03 \%$.

\section{Discussion}

In this paper we presented the findings of a naturalistic study that took place in a Crisis Intervention Center (CIC). In particular, we aimed to characterize the sociodemographical and clinical profiles of the patients referred to the CIC in a context of emotional crisis and participating in intensive treatment, as well as assessing the effectiveness of the latter. 
Table 5 Results of the comparisons between intake and discharge

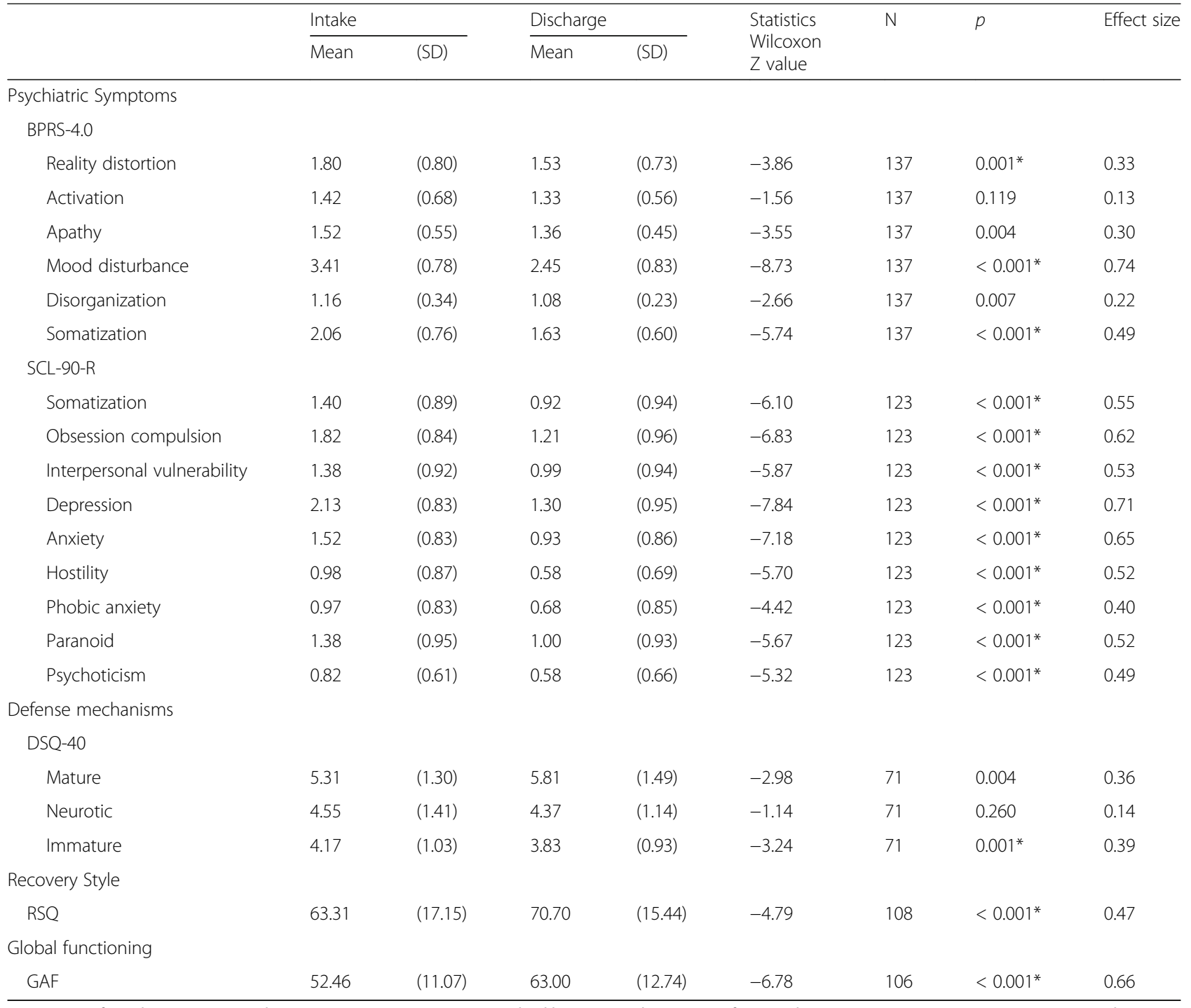

BPRS-4.0 Brief Psychiatric Rating Scale version 4.0, SCL-90-R Symptom Checklist - Revised, DSQ-40 Defense Style Questionnaire 40 items, RSQ Recovery Style Questionnaire, GAF Global Assessment Functioning

${ }^{*} p<0.05$ after Bonferroni correction for multiple comparison

\section{Sample characteristics at intake}

Patients were middle aged, with a higher proportion of women. The majority of patients was addressed to the $\mathrm{CIC}$ by the emergency unit of the hospital or by private practitioners which is in accordance with previous findings $[15,22,50,51,53]$, because the patients had attempted to commit suicide, had suicidal ideation or presented depressed mood due to relational difficulties either in their family, in their couple or at their work place. Most of the patients were diagnosed as presenting a depressive or neurotic disorder with low global functioning and were administered a polypharmacological treatment. Regarding socio-demographic variables and crisis features, our findings are consistent with those reported in previous studies conducted in very similar psychiatric services providing crisis treatment $[9,14,15,17,50-53]$. Nevertheless, we found a higher percentage of working patients and student patients than in other studies [22] as well as patients on average 10 years older than those from Marini's study [50]. Moreover, unlike previous studies [9, 23, 52], our sample almost did not include patients with schizophrenia and related psychotic disorders. As previously observed, the psychiatric symptoms are severe $[9,15,23,50]$. As none of the previous studies assessed the defense mechanisms in patients with emotional crisis, we cannot compare our results and we can only state that at the beginning of the treatment patients used preferentially mature defense mechanisms, followed by neurotic and immature defense mechanisms. Regarding the recovery styles, the integration style was common in patients with affective disorders, 
Table 6 Clinical evolution using the reliability change index

\begin{tabular}{|c|c|c|c|c|c|c|}
\hline & \multicolumn{2}{|c|}{ Deteriorated } & \multicolumn{2}{|c|}{ Undetermined } & \multicolumn{2}{|c|}{ Improved } \\
\hline & $\mathrm{N}$ & (\%) & $\mathrm{N}$ & (\%) & $\mathrm{N}$ & (\%) \\
\hline \multicolumn{7}{|l|}{ Psychiatric symptoms } \\
\hline \multicolumn{7}{|l|}{ BPRS-4.0 } \\
\hline Reality distortion & 5 & $(3.65)$ & 132 & (96.35) & 0 & $(0.00)$ \\
\hline Activation & 5 & $(3.65)$ & 122 & $(89.05)$ & 10 & $(7.30)$ \\
\hline Apathy & 3 & $(2.19)$ & 124 & $(90.51)$ & 10 & $(7.30)$ \\
\hline Mood disturbance & 5 & $(3.65)$ & 62 & $(45.26)$ & 70 & $(51.09)$ \\
\hline Disorganization & 11 & $(8.03)$ & 123 & (89.78) & 3 & $(2.19)$ \\
\hline Somatization & 3 & $(2.19)$ & 110 & $(80.29)$ & 24 & $(17.52)$ \\
\hline \multicolumn{7}{|l|}{ SCL-90-R } \\
\hline Somatization & 15 & $(12.20)$ & 70 & $(56.91)$ & 38 & (30.89) \\
\hline Obsession compulsion & 1 & $(0.81)$ & 79 & $(64.23)$ & 43 & (34.96) \\
\hline Interpersonal vulnerability & 5 & $(4.07)$ & 79 & $(64.23)$ & 39 & (31.71) \\
\hline Depression & 8 & $(6.50)$ & 51 & $(41.46)$ & 64 & (52.03) \\
\hline Anxiety & 3 & (2.59) & 64 & $(55.17)$ & 49 & $(42.24)$ \\
\hline Hostility & 2 & $(1.77)$ & 77 & $(68.14)$ & 34 & (30.09) \\
\hline Phobic anxiety & 7 & (5.69) & 95 & $(77.24)$ & 21 & $(17.07)$ \\
\hline Paranoid & 1 & $(0.81)$ & 99 & $(80.49)$ & 23 & $(18.07)$ \\
\hline Psychoticism & 3 & $(2.65)$ & 61 & (53.98) & 49 & $(43.36)$ \\
\hline \multicolumn{7}{|l|}{ Defense mechanisms } \\
\hline \multicolumn{7}{|l|}{ DSQ-40 } \\
\hline Mature & 5 & (7.04) & 44 & $(64.79)$ & 20 & $(28.17)$ \\
\hline Neurotic & 13 & $(18.31)$ & 46 & $(67.61)$ & 10 & (14.08) \\
\hline Immature & 13 & $(18.31)$ & 50 & $(73.24)$ & 6 & $(8.45)$ \\
\hline \multicolumn{7}{|l|}{ Recovery Style } \\
\hline RSQ & 7 & $(6.48)$ & 55 & (58.33) & 38 & (35.19) \\
\hline \multicolumn{7}{|l|}{ Global functioning } \\
\hline GAF & 4 & $(3.70)$ & 65 & $(60.19)$ & 39 & $(36.11)$ \\
\hline
\end{tabular}

BPRS-4.0 Brief Psychiatric Rating Scale version 4.0, SCL-90-R Symptom Checklist - Revised, DSQ-40 Defense Style Questionnaire 40 items, RSQ Recovery Style Questionnaire, GAF Global Assessment Functioning

which is in accordance with previous observations [54]. These last two observations seem contrary to the psychodynamic theory of crisis [27] and suggest that emotional crisis does not have the deleterious impact expected on psychological functioning.

\section{Dropouts and missing data}

The attrition rate and missing data were high. The proportion of dropouts found herein fell in the upper range, that is $15 \%$ to $60 \%$, of premature termination reported in the literature [55]. However, previous studies conducted in similar crisis centers reported lower rates of premature termination, i.e. from $16 \%$ to $37.5 \%[14,50,51]$. This difference may be explained by the fact that patients having already started to work before the end of the crisis intervention may have found irrelevant to come back to the CIC for research purposes. Nevertheless, no significant difference between attender and non-attender groups was found and, missing data having mostly occurred at random, we can assume that drop-outs and missing data have only slightly biased effects on the findings [49].

\section{Crisis intervention efficiency}

The crisis intervention lasted on average 8 weeks, but there was a considerable variation among patients, ranging from 2 to 58 weeks. This variation likely occurred because of the complexity of the cases associating psychiatric symptom severity and social problems (e.g. homelessness or dismissal occurring during intervention...).

Findings of this study suggest effectiveness on different levels of a time-limited intensive psychodynamic 
oriented crisis intervention. First, in terms of symptom reduction, a change of the defense mechanisms towards more mature ones was found, possibly as a better integration of the crisis as well as an improvement of the general functioning occurred. However, the effect size and reliable change index tone down a bit the high statistical differences observed after the crisis intervention. Symptom reduction and improvement in social functioning are in accordance with the findings of previous studies underwent in care units including aspects of crisis intervention [15, 24, 25, 52]. Nevertheless, to the best of our knowledge, this is the first study demonstrating a significant change of the predominant defenses mechanisms and change in recovery style; therefore, comparisons with other studies were not possible.

\section{Scope of the findings for clinical practice}

The results reported here might have important clinical implications. The socio-demographic and clinical profile of the patients at intake in a Crisis center may help the clinician to offer the patients tailored pharmacological and psychological (e.g. individual or group interventions) treatments. They also provide evidence that crisis intervention should not only address psychiatric symptoms - especially suicidal thoughts and behavior - and psychopathology in general, but also promote psychological adjustment to the emotional crisis (e.g. increasing mature defense mechanisms and integration recovery style) as well as social functioning and prevent future decompensations. They also support the view that family and couple psychotherapy should also be considered as crucial ingredients in crisis intervention. In addition, it is important to note that patients may benefit from a specific treatment approach according to their recovery style [41, 56-59].

\section{Limitations}

The generalization of our findings regarding crisis intervention effectiveness is restricted due to several limitations that need to be acknowledged. The naturalistic design of the study did not allow to control whether improvements were due to the natural course of emotional crisis resolution. The high percentage of dropouts suggested that psychodynamically oriented crisis intervention is not suitable for all patients. Moreover, we could not differentiate crisis intervention dropouts (patients terminating prematurely the intervention) from study dropouts (patients participating in the intervention but withdrawing from the study). The length of intervention was neither defined nor controlled for. Only patients entering the intensive crisis program and willing to participate in the study were included. Thus, it is possible that the profile of these patients may be different to the one of the patients addressed to the CIC. It is also to be mentioned that psychiatric diagnosis was not established using a structured interview such as the SCID [60] or MINI [61, 62]. Moreover, the clinical assessments used did not take into account aversive past life events, which could be related to several psychiatric disorders and considered as a pivotal ingredient in the current emotional crisis [63-65]. Comorbidities (e.g., severe medical conditions, substance abuse) were not considered. Moreover, follow-up assessment at several time points (e.g. 6 and 12 months), which is common in psychotherapy research $[66,67]$, was not used. Another limitation is the absence of differentiation between first and multiple episode crises which probably need different interventions [17]. As there are no normative data for the general population regarding several instruments (e.g. BPRS 4.0, DSQ-40, RSQ, and GAF), Clinical Significance could not be applied [46], and thus the clinical outcome was not fully examined. Moreover, the impact of staff members' framework and adherence to rigorous crisis concepts was not considered. Finally, adverse effects occurring during intervention (e.g. suicide attempts, suicide, scarification, hospitalization, violent behaviours...) were not recorded.

\section{Conclusion}

In summary, patients facing an emotional crisis do not form a homogenous group, regarding e.g. crisis context, diagnosis, symptom severity. As a consequence, specific brief family therapy, couple therapy or suicide intervention should be viewed as adjunctive treatments for most of the individuals referred to a crisis intervention center. This also has implications for crisis intervention staff members who may benefit from continuous supervision and specific training (e.g. suicide intervention skills training). This study suggests that brief psychodynamicallyoriented crisis interventions in specific units like the CIC may contribute to alleviate symptoms, to change defense mechanisms, as patients present more mature defenses mechanisms after treatment, to increase global functioning, and to enhance the patients' psychological adjustment to their emotional crisis towards a more "integrative" recovery style.

Findings reported herein provide a basis for our future work on the generalization of the study to other crisis centers and on the effectiveness of brief intensive crisis treatment. Further research is needed to clarify whether the improvements are clinically significant and remain stable on the long run.

\section{Additional file}

Additional file 1: Table: Comparisons between Admission Assessment Non-Attender and Attender Groups. (DOCX 18 kb)

\section{Abbreviations}

BPRS 4.0: Brief psychiatric rating scale version 4.0; BPRS 4.0-C: Brief psychiatric scale version 4.0 - categories; CIC: Crisis intervention center; DSQ 40: Defense style questionnaire; EMDR: Eye movement desensitization and reprocessing; 
ES: Effect size; GAF: Global assessment functioning; ICD-10: International Classification of Diseases, 10th revision; M: Mean; MINI: Mini International Neuropsychiatric Interview; RCI: Reliable change index; RSQ: Recovery style questionnaire; SARS: Severe acute respiratory syndrome; SCID: Structural clinical interview for DSM; SCL-90-R: Symptom checklist 90 revised; SCL-90-R-C: Symptom checklist 90 revised - categories; SD: Standard deviation; SE: Standard error; SPSS: Statistical package for social sciences; $X$ : Patients scores

\section{Acknowledgments}

We would like to thank the patients for willing to participate to the study despite facing a difficult moment in their lives.

\section{Funding}

This research was not supported by public or private funds or grants.

\section{Availability of data and materials}

Additional information about data set and statistical analyses are available on request.

\section{Authors' contributions}

AZ designed the study, wrote the protocol, examined the patients, analysed the data, wrote the draft of the paper and selected the references. LB examined the patients, contributed to the data base, and corrected and edited the final version of the paper. JPB contributed to the study protocol, supervised the data collection and reviewed the final manuscript. All authors read and approved the final manuscript.

\section{Competing interests}

The authors declare that they have no competing interests.

\section{Consent for publication}

Not applicable.

\section{Ethics approval and consent to participate}

The local Ethics Committee of the University Hospitals of Geneva (Commission Centrale d'Ethique de la recherche sur l'être humain, CCER/HUG) approved the study protocol (protocol number Psy 05-017). All patients were informed of the purpose and procedure of the study prior to giving their written informed consent to participate. Assessments were in accordance with the Helsinki declaration.

\section{Authors' note}

Parts of this study were presented as posters at the 13th SSP Congress, 10-12 September 2014, Basel (Switzerland) and at the 13th "L'Encéphale" Congress, 21-23 January 2015, Paris (France).

\section{Publisher's note}

Springer Nature remains neutral with regard to jurisdictional claims in published maps and institutional affiliations.

\section{Author details}

'Department of Mental Health and Psychiatry, University Hospitals of Geneva, Geneva, Switzerland. ${ }^{2}$ Department of Psychiatry, University of Lausanne, Lausanne, Switzerland. ${ }^{3}$ HUG Département de Santé Mentale et de Psychiatrie, Site Belle-ldée, Ch. du Petit-Bel-Air 2, CH-1225 Chêne-Bourg, Switzerland

Received: 15 October 2016 Accepted: 30 March 2017

Published online: 07 April 2017

\section{References}

1. James RK, Gilliland BE. Crisis intervention strategies. 7th ed. Belmont: Brooks/ Cole; 2013.

2. Roberts AR, Everly GS. A meta-analysis of 36 crisis intervention studies. Brief Treat Crisis Interv. 2006;6:10-21.

3. Marjanovic Z, Greenglass ER, Coffey S. The relevance of psychosocial variables and working conditions in predicting nurses' coping strategies during the SARS crisis: an online questionnaire survey. Int Nurs Stud. 2007;44:991-8.

4. Roberts AR, Yeager KR. Pocket guide to crisis intervention. New-York: Oxford University Press; 2009.
5. Roberts AR. Bridging the past and present to the future of crisis intervention and crisis management. In: Roberts AR, editor. Crisis intervention handbook: Assessment, treatment and research. 3rd ed. New York: Oxford University Press; 2005. p. 3-33.

6. Rosen A. Crisis management in the community. Med J Aust. 1997;167(11-12):633-8.

7. Everly GS, Mitchell JT. Critical incident stress management (CISM): a new era and standard of care in crisis intervention. Ellicott City: MD: Chevron Publishing; 1999.

8. Loughran H. Understanding crisis therapies. London and Philadelphia: Jessica Kingsley Publishers; 2011.

9. Johnson S, Nolan F, Pilling S, Sandor J, Hoult J, McKenzie N, White IR, Thompson M, Bebbington P. Randomized controlled trial of acute mental health care by a crisis resolution team: the north Islington crisis study. BMJ. 2005;331:599-603

10. Wheeler C, Lloyd-Evans B, Churchard A, Fitzgerald C, Fullarton K, Mosse L, Paterson B, Zugaro CG, Johnson S. Implementation of the crisis resolution team model in adult mental health settings: a systematic review. BMC Psychiatry. 2015;15:74. doi:10.1186/s12888-015-0441-x.

11. Howard LM, Leese M, Byford S, Killaspy H, Cole L, Lawlor C, Johnson S. Methodological challenges in evaluating the effectiveness of women's crisis houses compared with psychiatric wards. J Nerv Ment Dis. 2009;197:722-7.

12. Tschacher $W$, Jacobshagen $N$. Analysis of crisis intervention processes. Crisis. 2002:23(2):59-67. doi:10.1027//0227-5910.23.2.59.

13. Andreoli A, Lalive J, Garrone G. Crise et intervention de crise en psychiatrie. Lyon: SIMEP; 1986

14. Andreoli AV, Muehlebach A, Gognalons M, Abensur J, Grimm S, Frances AJ. Crisis intervention response and long-term outcome: a pilot study. Compr Psychiatry. 1992;33(6):388-96. doi:10.1016/0010-440X(92)90061-T.

15. Bacchetta JP, Zanello A, Varnier M, Stebler E, Safran E, Ferrero F, Bertschy G, Merlo MCG. Développement des centres de crise à Genève: Impact sur les hospitalisations. Schweiz Arch Neurol Psychiatr. 2009;160(3):116-21.

16. Glover G, Arts G, Babou KS. Crisis resolution/home treatment teams and psychiatric admission rates in England. Br J Psychiatry. 2006;189:441-5.

17. Sentissi O, Bartolomei J, Moeglin C, Baeriswyl-Cottin R. Rey- Bellet P. The Geneva model of crisis intervention: a retrospective study. Psychol Psychoter. 2014:4:149. doi:10.4172/2161-0487.1000150.

18. Cotton MA, Johnson S, Bindman J, Sandor A, White IR, Thornicroft G, Nolan F, Pilling S, Hoult J, McKenzie N, Bebbington P. An investigation of factors associated with psychiatric hospital admission despite the presence of crisis resolution teams. BMC Psychiatry. 2007;7(52). doi: 10.1186/1471-244X-7-52.

19. Comtois KA, Jobes DA, S O'Connor S, Atkins DC, Janis K, E Chessen C, Yuodelis-Flores C. Collaborative assessment and management of suicidality (CAMS): feasibility trial for next-day appointment services. Depress Anxiety. 2011;28(11):963-72.

20. Currier JM, Holland JM, Neimeyer RA. Do CBT-based interventions alleviate distress following bereavement? A review of the current evidence. Int J CognTher. 2010:3(1):77-93.

21. Sjølie H, Karlsson B, Kim HS. Crisis resolution and home treatment: structure, process, and outcome-a literature review. J Psychiatr and Ment Health Nurs. 2010;17(10):881-92.

22. Bartolomei J, Sentissi O. Baeriswyl-Cottin R, Rey-Bellet P. Centres de Thérapies Brèves (CTB) à Genève: la crise dans les centres de crise? Schweizer Archiv Neurol Psychiatr. 2011;162(4):161-166.

23. Johnson S. Crisis resolution and intensive home treatment teams. Psychiatry. 2007;6(8):339-42

24. Wernicke F, Stieglitz RD, Riecher-Rössler A. Stationäre Psychiatrische Kurzzeit-Krisenintervention am Basler Universitätsspital. Schweizer Archiv Neurol Psychiatr. 2010;161(1):17.

25. Reisch T, Schlatter P, Tschacher W. Efficacy of crisis intervention. Crisis. 1999;20(2):78-85.

26. Caplan G. Principles of preventive psychiatry. New York: Basic Books; 1964

27. De Coulon N. La crise. Stratégies d'intervention thérapeutique en psychiatrie. Paris: Gaëtan Morin éditeur; 1999.

28. Khawaja IS, Westermeyer JJ. Providing crisis-oriented and recovery-based treatment in partial hospitalization programs. Psychiatry (Edgmont). 2010;7(2):28-31.

29. Craig P, Dieppe P, Macintyre S, Michie S, Nazareth I, Petticrew M. Developing and evaluating complex interventions: the new Medical Research Council guidance. BMJ. 2008;337:979-83. https://doi.org/10.1136/bmj.a1655.

30. Berger ML, Martin BC, Husereau D, Worley K, Allen JD, Yang W, Crown W. A questionnaire to assess the relevance and credibility of observational 
studies to inform health care decision making: an ISPOR-AMCP-NPC good practice task force report. Value Health. 2014;17(2):143-56.

31. Ventura J, Lukoff D, Nuechterlein KH, Liermann RP, Green M, Shaner A. Appendix1: brief psychiatric rating scale (BPRS) expanded version (4.0) scales, anchor points, and administration manual. Int J Methods Psychiatr Res. 1993;3:227-44.

32. Derogatis LR. SCL-90: administration, scoring and procedure manual-1 for the $R$ (revised) version. Baltimore: John Hopkins University School of Medecine; 1977.

33. Fortin MF, Coutu-Wakulczyk G. Validation et normalisation d'une mesure de santé mentale: le SCL-90-R. Rapport présenté au Conseil québécois de la recherche sociale (CQRS). Université de Montréal: Faculté des sciences infirmières; 1985.

34. Brenner HD, Merlo MCG. Definition of therapy-resistant schizophrenia and its assessment. Eur Psychiatry. 1995;10:11s-7s.

35. Zanello A, Berthoud L, Ventura J, MCG M. The brief psychiatric rating scale (version 4.0) factorial structure and its sensitivity in the treatment of outpatients with unipolar depression. Psychiatry Res. 2013;210:626-33.

36. Franke G. Die symptom Chekliste von LR Derogatis - manual zur deutsche version [SCL-90-R. The symptom Cheklist by LR Derogatis - manual for the German version] $2^{\text {nd }}$ ed. Göttingen: Beltz; 2002.

37. Heaton R, Paulsen JS, McAdams LA, Kuck J, Zisook S, Braff D, Harris JM, Jeste DV. Neuropsychological deficits in schizophrenics: relationship to age, chronicity, and dementia. Arch Gen Psychiatry. 1994:51(6):469-76.

38. Andrews $\mathrm{G}$, Singh $\mathrm{M}$, Bond $\mathrm{M}$. The defense style questionnaire. J Nerv Ment Dis. 1993;181(4):246-56.

39. Guelfi JD, Hatem N, Damy C, Corruble E. Intérêt du Defense Style Questionnaire (DSQ), questionnaire sur les mécanismes de défense. Ann Med Psychol. 2000;158:594-601.

40. Drayton M, Birchwood M, Tower P. Early attachment experience and recovery from psychosis. Br J Clin Psychol. 1998;37:269-84.

41. McGlashan TH, Levy ST, Carpernter WT. Integration and sealing-over: clinically distinct recovery styles from schizophrenia. Arch Gen Psychiat. 1975;32:1269-72.

42. Mulligan A, Lavender T. An investigation into relationship between attachment, gender and recovery from psychosis in stable community-based sample. Clin Psychol Psychot. 2010;17:269-84.

43. American Psychiatric Association. Diagnostic and Statistical Manual of Mental Disorders, $4^{\text {th }}$ ed. (DSM-IV). Washington DC: APA; 1994.

44. Pallant J. SPSS survival manual: a step-by-step guide to data analysis using SPSS version 15. Nova lorque: McGraw Hill; 2007.

45. Cohen J. Statistical power analyses for the social sciences. Hillsdale: Lawrence Erlbaum; 1988.

46. Jacobson NS, Truax P. Clinical significance: a statistical approach to defining meaningful change in psychotherapy research. J Consult Clin Psychol. 1991;59(1):12-9.

47. Wilkinson L. Task force on statistical inference, American Psychological Association, science directorate. Statistical methods in psychology journals: guidelines and explanations. Am Psychol. 1999;54(8):594-604.

48. Schlomer GL, Bauman S, Card NA. Best practices for missing data management in counseling psychology. J Couns Psychol. 2010;57(1):1-10.

49. Fewtrell MS, Kennedy K, Singhal A, Martin RM, Ness A, Hadders-Algra M, Lucas A. How much loss to follow-up is acceptable in long-term randomised trials and prospective studies? Arch Dis Child. 2008;93(6):458-61.

50. Marini M, Sermanzin M, Vignaga F, Gardiolo M, Drago A, Caon F, Benetazzo M, Pavan C, Piotto A, Frederico L, Corinto B, Pavan L. Dropout in institutional emotional crisis counseling and brief focused intervention. Brief Treat Crisis Interv. 2005;5:356-67.

51. Henzen A, Moeglin C, Giannakopoulos P, Sentissi O. Determinants of dropout in a community-based mental health crisis centre. BMC Psychiatry. 2016;16(1):1-7

52. Adams CL, El-Mallakh RS. Patient outcome after treatment in a communitybased crisis stabilization unit. J Behav Health Serv Res. 2009;36(3):396-9.

53. Hasselberg N, Grawe RW, Johnson S, Ruud T. An implementation study of the crisis resolution team model in Norway: are the crisis resolution teams fulfilling their role? BMC Health Ser Res. 2011;11(1):1-9.

54. Thompson KN, McGorry PD, Harrigan SM. Recovery style and outcome in first-episode psychosis. Schizophr Res. 2003;62:31-6.

55. Chiesa $\mathrm{M}$, Drahorad C, Longo $\mathrm{S}$. Early termination of treatment in personality disorder treated in a psychotherapy hospital. Br Psychiatry. 2000;177(2):107-11.

56. McGlashan TH, Levy ST. Sealing-over in a therapeutic community. Psychiatry. 1977;40:55-65
57. Jackson H, McGorry P, Henry L, Edwards J, Hulbert C, Harrigan S, Dudgeon P, Francey S, Maude D, Cocks J, Power P. Cognitively oriented psychotherapy for early psychosis (COPE): a 1-year follow-up. Brit J Clin Psychol. 2001;40:57-70.

58. McGlashan TH. Recovery style from mental illness and long-term outcome. J Nerv Ment Dis. 1987;175:681-5.

59. Startup M. Cognitive behavior therapy and recovery from acute psychosis: case studies of two contrasting styles. J Contemp Psychother. 2006;36(1). doi:10.1007/s10879-005-9002-9.

60. First MB, Williams JBW, Karg RS, Spitzer RL. Structured clinical interview for DSM-5 disorders, clinician version (SCID-5-CV). Arlington: American Psychiatric Association; 2015

61. Lecrubier Y, Sheehan D, Weiller E, Amorim P, Bonora I, Sheehan K, Janavs J, Dunbar G. The M.I.N.I. International neuropsychiatric interview (M.I.N.I.) a short diagnostic structured interview: reliability and validity according to the CIDI. Eur Psychiat. 1997:12:224-31.

62. Sheehan DV, Lecrubier Y, Harnett-Sheehan K, Janavs J, Weiller E, Bonara LI, Keskiner A, Schinka J, Knapp E, Sheehan MF, Dunbar GC. Reliability and validity of the M.I.N.I. International neuropsychiatric interview (M.I.N.I.): according to the SCID-P. Eur Psychiat. 1997;12:232-41.

63. Klein J, Roniger A, Schweiger U, Spath C, Brodbeck J. The association of childhood trauma and personality disorders with chronic depression: a cross-sectional study in depressed outpatients. J Clin Psychiatry. 2015;76(6):794-801. doi:10.4088/JCP.14m09158.

64. Mandelli L, Petrelli C, Serretti A. The role of specific early trauma in adult depression: a meta-analysis of published literature. Childhood trauma and adult depression. Eur Psychiat. 2015;20:665-80.

65. Norman R, Byambaa M, De R, Butchart A, Scott J, Vos T. The long-term health consequences of child physical abuse, emotional abuse and neglect: a systematic review and meta-analysis. PLoS Med. 2012;9(11):e1001349. doi:10.1371/journal.pmed.1001349.

66. Ambresin G, Despland JN, Preisig M, de Roten Y. Efficacy of an adjunctive brief psychodynamic psychotherapy to usual inpatient treatment of depression: rationale and design of a randomized controlled trial. BMC Psychiatry. 2012;12(1):1-9.

67. Semerari A, Cucchi M, Dimaggio G, Cavadini D, Carcione A, Battelli V, Nicolò G, Pedone R, Siccardi T, D'Angerio S, Ronchi P, Maffei C, Smeraldi E. The development of the Metacognition Assessment interview: instrument description, factor structure and reliability in a non-clinical sample. Psychiatry Res. 2012;200(2):890-5.

\section{Submit your next manuscript to BioMed Central and we will help you at every step:}

- We accept pre-submission inquiries

- Our selector tool helps you to find the most relevant journal

- We provide round the clock customer support

- Convenient online submission

- Thorough peer review

- Inclusion in PubMed and all major indexing services

- Maximum visibility for your research

Submit your manuscript at www.biomedcentral.com/submit 